\title{
Seroprevalence of Dengue Infection in Patients Suffering from Acute Febrile Illness in a Tertiary Care Centre-Time to Introduce Dengue Vaccine CYD-TDV to Susceptible Population
}

\author{
Nidhi Prasad ${ }^{1}$, Vidyut Prakash ${ }^{1 *}$, Keshav Kumar Bimal ${ }^{2}$ and S.K. Shahi ${ }^{2}$ \\ ${ }^{1}$ Department of Virology, IGIMS, Patna, Bihar, India \\ ${ }^{2}$ Department of Microbiology, IGIMS, Patna, Bihar, India \\ *Corresponding author
}

\begin{abstract}
A B S T R A C T
Dengue, an important vector-borne viral disease and one of the most common causes of acute febrile illness (AFI), is considered as a notable public health problem because it spreads rapidly by the bite of infected mosquito and has high incidence of mortality,

Keywords

Acute febrile illness

(AFI), Dengue, Dengue

vaccine CYD-TDV,

Vector borne viral disease

Article Info

Accepted:

06 May 2018

Available Online:

10 June 2018 morbidity and economical challenges associated with it. Bihar, having a population of 104, 099, 452, average annual rainfall of $1,186 \mathrm{~mm}$ and having a breeding place for vector Aedes, is prone to dengue outbreaks. Recently, the first dengue vaccine, Dengvaxia (CYD-TDV) has been introduced. Objective of the study is to determine seroprevalence of dengue infection in patients suffering from AFI, seroprevalence of susceptible population and to ascertain whether it is appropriate time to introduce dengue vaccine CYD-TDV to susceptible population. A retrospective cross sectional observational study, of records of all patients of AFI, whose sera was subjected to simultaneous detection of dengue NS1 antigen, antidengue IgM and IgG antibody was done from July 2016 to July 2017 at a tertiary care center in eastern India. Out of 384 samples tested, a total of 38 (9. 9\%) samples tested positive for dengue infection and the remaining, i.e. 346/384 (90. 20\%) were negative and the patients were considered susceptible to dengue infection. The seroprevalence of dengue was high especially in post-monsoon period. Besides continuing preventive and control measures, authorities should consider introduction of the dengue vaccine CYD-TDV.
\end{abstract}

\section{Introduction}

Acute febrile illness (AFI) is a major cause of morbidity and mortality (Robinson et al., 2018). Dengue, an important vector-borne viral disease, and one of the most common causes of AFI, is considered as a notable public health problem because it is spread rapidly by the bite of infected mosquito and there the high incidence of mortality, morbidity and economical challenges associated with it (World Health Organization, 2009). The three main factors responsible for spread of dengue infection are: dengue virus, Aedes mosquito (vector) and environment favouring breeding of mosquitoes. Dengue virus has four different serotypes DENV-1, DENV-2, DENV-3, DENV-4. Human infection with one serotype provides life-long immunity to the individual against that specific serotype, but does not provide immunity against other serotypes. On the 
contrary, due to cross-reactive antibodies it enhances the severity of disease caused by subsequent infection by other serotypes. Thus, in dengue endemic countries, people are likely to be infected more than once over their lifetime with more severe disease after a secondary infection with a heterologous serotype (WHO, 2018; Halstead, 2007; Martina et al., 2009). In 2016, there were reports of co-circulation of all the four dengue virus serotypes in India (Shrivastava et al., 2018). Although various steps have been taken at household, community and government levels to control dengue, but its global incidence has grown dramatically in recent decades and is estimated to cause 50 to 100 million cases of Acute febrile illness (AFI) every year (Halstead, 2007; Brady et al., 2012; Chakravarti et al., 2012; Brady et al., 2012; Bhatt et al.,). What is of more alarming significance, is that many dengue infection cases present with severe disease leading to death. In 2015, out of 2.35 million dengue cases reported from Americas alone, 10, 200 cases were diagnosed as cases of severe dengue infection resulting in 1181 deaths. In 2016, there were reports of large dengue outbreaks throughout the world resulting in many deaths (WHO, 2018). The main emphasis for preventing or controlling dengue at present is to combat vector mosquitoes. Recently, the first dengue vaccine, Dengvaxia (CYD-TDV), by Sanofi Pasteur, has been introduced. It is alive recombinant tetravalent 3 dose dengue vaccine which is administered as $0,6,12$ months schedule. It was introduced in late 2015 and early 2016 and was registered for use in individuals 9-45 years of age living in endemic areas in several countries. WHO recommends that only those countries where epidemiological data indicate a high burden of dengue disease, should consider introduction of the dengue vaccine CYD-TDV.

Bihar, having a population of 104, 099, 452 and average annual rainfall of 1,186 $\mathrm{mm}$ and having a breeding place for vector Aedes, is prone to dengue outbreaks. To the best of our knowledge every few studies have been done in this part of Bihar to evaluate the seroprevalence of dengue infection amongst AFI cases especially after the availability of dengue vaccine.

Keeping this in mind we decided to do this study with the aim to find out the seroprevance of dengue infection in patients suffering from Acute febrile illness, seroprevalence of dengue infection negative but susceptible population in a tertiary care centre and to ascertain whether it is appropriate time to introduce dengue vaccine CYD-TDV to the susceptible population.

\section{Materials and Methods}

\section{Ethical approval}

Ethical approval from the Institutes Ethical committee was obtained for the study

\section{Study area}

The data was collected from the virology lab of a tertiary care centre in Eastern India which provides services to the local community as well as for other population coming from different regions of Bihar.

\section{Study design}

A retrospective cross sectional observational study of all the consecutive records of patients suffering from AFI, whose serum was subjected to simultaneous detection of dengue NS1 antigen, anti-dengue $\operatorname{IgM}$ and $\operatorname{IgG}$ antibody test from July 2016 to July 2017 was done.

\section{Inclusion criteria}

Records of patients, presenting with symptoms of fever for more than five days duration, with more than or equal to two of the following: 
joint pain, rash, myalgia, retro-orbital pain, headache and haemorrhagic manifestation and subjected to simultaneous detection of dengue NS1antigen, anti- dengue $\operatorname{IgM}$ and $\operatorname{IgG}$ antibody test by solid phase ICT (SDBIOLINE) from July 2016 to July 2017, were included in the study.

\section{Intervention that was done:}

The records were obtained and data was analyzed. The patient whose sera was found to be positive for either or all of the three parameters, i.e. IgG, IgM and NS1 was considered to be positive for dengue infection, either current or past infection. The patient whose sera was found to be negative for all three parameters, i.e. IgG, IgM and NS1 was considered to be negative for dengue infection but susceptible to dengue infection. Primary dengue was identified when either NS1 antigen or IgM was positive with a negative IgG test, while secondary dengue was diagnosed when either of the above was associated with IgG positivity.

\section{Results and Discussion}

Out of 384 samples tested, a total of 38 (9. $9 \%$ ) samples were tested positive for either one or more of the three markers i.e. NS1 Ag, $\operatorname{IgM}$ and $\operatorname{IgG}$ antibody tested. The remaining samples, i.e. $346 / 384$ (90. 20\%) tested negative for all the three markers.

Out of the 38 serum positive samples, 20 (52. $63 \%$ ) patients were positive for NS1 Ag only, $6(15.78 \%)$ positive for IgM only, while 1 (2. $6 \%$ ) were positive for IgG only. More than one marker was detected in the remaining 11 (28. 94\%) samples.

Maximum number of samples for testing of dengue infection was received in the postmonsoon period, i.e. in the month of September, October and November.
Maximum number of serologically positive cases were also found in the month of September $(11 / 81=13.5 \%)$, October $(11 / 81=$ 13. $5 \%)$ and November $(6 / 74=10.81 \%)$. Though dengue was present throughout the year there was significant dip in the receipt of sample and sample testing positive for dengue infection in the month from January to June.

Primary dengue was detected in $33(86.84 \%)$ cases and secondary dengue infection was detected in $5(13.16 \%)$ cases.

In this retrospective cross sectional observational study, in which records of patients suffering from AFI whose serum was subjected to simultaneous detection of dengue NS1antigen, anti- dengue $\operatorname{IgM}$ and $\operatorname{IgG}$ antibody test by solid phase ICT (SDBIOLINE) from July 2016 to July 2017 was studied and analyzed. The patient whose sera was found to be positive for either or all of the three parameters, i.e. IgG, IgM and NS1 was considered to be positive for dengue infection, whether current or past infection. The patient whose sera was found to be negative for all three parameters, i.e. $\operatorname{IgG}, \operatorname{IgM}$ and NS1 was considered to be negative for dengue infection and susceptible to dengue infection. Primary dengue was identified when either NS1 antigen or IgM was positive with a negative $\operatorname{IgG}$ test, while secondary dengue was diagnosed when either of the above was associated with IgG positivity.

In this study we found that, out of 384 samples, a total of $38(9.9 \%)$ samples tested positive for either one or more of the three markers i.e. NS1Ag, IgM and IgG antibody. Out of the 38 positive serum samples, 20 (52. $63 \%$ ) were positive for NS1Ag only, 6 (15. $78 \%$ ) were positive for IgM only, while1 (2. $6 \%$ ) sample were positive for IgG only. More than one marker was detected in the remaining 11 (28. 94\%) samples. Maximum number of samples for testing of dengue infection was 
received in the post-monsoon period, i.e. in the month of September, October and November. Maximum number of serologically positive cases were also found in the month of September $(11 / 81=13.5 \%)$, October $(11 / 81=$ $13.5 \%)$ and November $(6 / 74=10.81 \%)$.

Though dengue was present almost throughout the year, there was a significant dip in the receipt of sample and sample testing positive for dengue infection in the month from January to June. Primary dengue was detected in $33(86.84 \%)$ cases and secondary dengue infection was detected in $5(13.16 \%)$ cases. The dengue infection has been reported earlier from various regions of India including Nagpur (Rodrigues et al., 1972), Parbhani and Dhulein Maharashtra (Mehendale et al., 1991; Padbidri et al., 1996), Bangalore (George and Soman, 1975) Punjab (Kaur et al., 1997) and Delhi (Gupta et al., 2005). In the present study, maximum number of dengue cases occurred during the post-monsoon season, i.e., from September to November only, which is similar to most of the previous outbreaks in India (Kaur et al., 1997; Gupta et al., 2005).

Table.1 Seroprevalence of dengue infection positive and negative cases
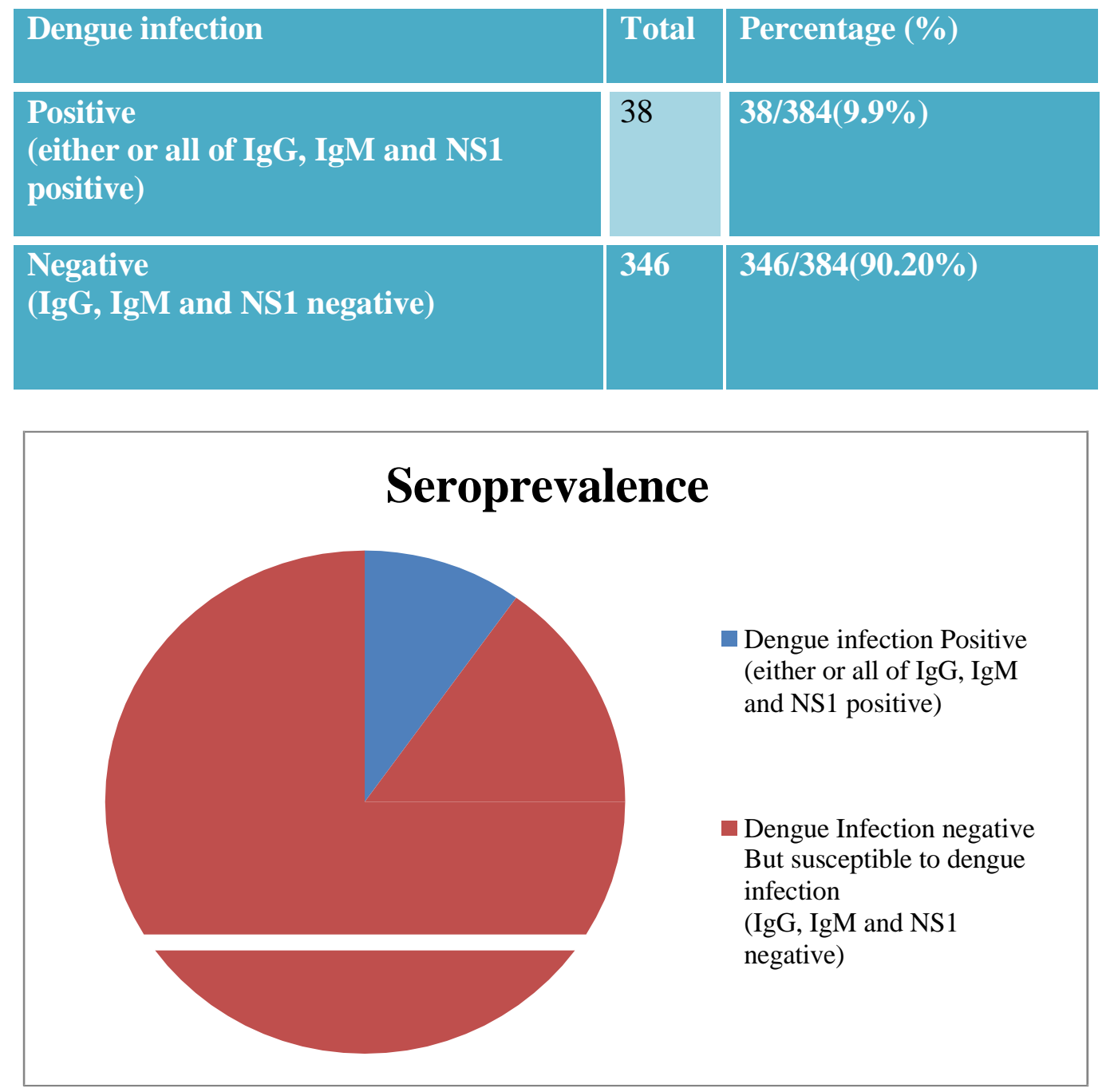
Table.2 Distribution of various dengue specific parameters

\begin{tabular}{|l|l|l|}
\hline Parameter & Total & Percent (\%) \\
\hline Only IgM positive & 6 & $\mathbf{1 5 . 7 8 \%}$ \\
\hline Both NS1 and IgM positive & 7 & $\mathbf{1 8 . 4 2 \%}$ \\
\hline Only IgG positive & 1 & $\mathbf{2 . 6 \%}$ \\
\hline IgG and NS1 positive & 1 & $\mathbf{2 . 6 \%}$ \\
\hline IgG and IgM positive & 1 & $\mathbf{2 . 6 \%}$ \\
\hline IgG, IgM and NS1 positive & 2 & $\mathbf{5 . 2 \%}$ \\
\hline Only NS1 positive & 20 & $\mathbf{5 2 . 6 3 \%}$ \\
\hline Seropositivity & 38 & $\mathbf{9 . 5 7 \%}$ \\
\hline
\end{tabular}

Chart.1 Showing month wise distribution of total sample received and number of seropositive case

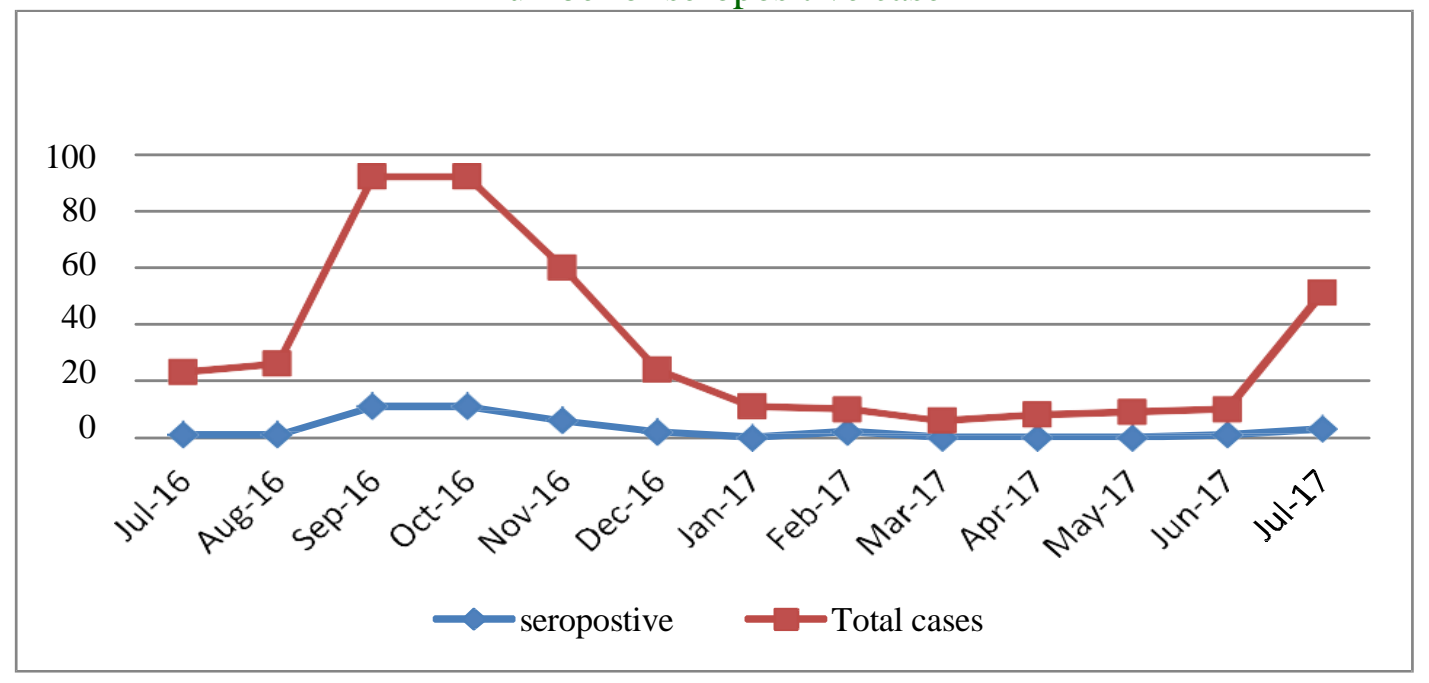

Table.3 Showing distribution of primary and secondary dengue infection

\begin{tabular}{|l|l|l|}
\hline Parameter & Total & Percent (\%) \\
\hline OnlyNS1positive & $\mathbf{2 0}$ & $\mathbf{5 2 . 6 3 \%}$ \\
\hline OnlyIgMpositive & 6 & $\mathbf{1 5 . 7 8 \%}$ \\
\hline BothNS1IgMpositive & 7 & $\mathbf{1 8 . 4 2 \%}$ \\
\hline PrimaryInfection & $\mathbf{3 3}$ & $\mathbf{8 6 . 8 4 \%}$ \\
\hline OnlyIgGpositive & 1 & $\mathbf{2 . 6 \%}$ \\
\hline IgGandNS1 positive & 1 & $\mathbf{2 . 6 \%}$ \\
\hline IgG and IgM positive & 1 & $\mathbf{2 . 6 \%}$ \\
\hline IgG, IgM and NS1positive & 2 & $\mathbf{5 . 2 \%}$ \\
\hline Secondary Infection & 5 & $\mathbf{1 3 . 1 6 \%}$ \\
\hline
\end{tabular}


In the present study, out of 384 samples were tested, a total of 38 (9. 9\%) samples tested positive for dengue infection and the remaining sample, i.e. 346/384 (90.20\%) though negative. The patients with sera negative for dengue infection were considered susceptible to dengue infection in future. The study also indicated that the seroprevalence of dengue is still high especially in post-monsoon period.

Since the epidemiological data indicate a high burden of dengue disease, authorities should consider introduction of the dengue vaccine CYD-TDV especially during pre-monsoon and post-monsoon period. Preventive measures at household, community and government levels should also continue side by side to control dengue infection and all patients suffering from AFI should be subjected to dengue virus screening to initiate necessary management.

\section{Strength of the study}

To the best of our knowledge very few studies have been done in this part of Bihar to evaluate the seroprevalence of dengue infection amongst AFI cases especially after the availability of dengue vaccine. In this context, the current study becomes highly relevant as it gives a platform for future investigation to ascertain whether it is appropriate time to introduce dengue vaccine CYD-TDV to susceptible population.

\section{Limitations of the study}

This was a small study done only at one center based only on the serology records of virology lab at a tertiary care center of eastern India. Since strong antibody cross reactivity exists among the flavi virus family, so confirmation should be done by RT-PCR in human sera/plasma during first 5 days of suspected dengue infection. In this study, we could not trace the actual confirmed cases to find the actual prevalence of the diseases, actual prevalence of susceptible population, actual prevalence of primary and secondary dengue cases nor could we as certain the serotype prevalent in this part of Eastern India. We could not correlate between concurrent infection of dengue with more than one serotype and severity of the disease symptoms.

\section{Future research implicated}

Recently, increasing number of DHF and DSS cases are being reported in India, which may be either due to secondary infection with a different serotype or may be due to concurrent dengue virus (DENV) infections with different serotypes. Therefore, accurate determination of the occurrence of DENV serotype infection and co-infections should be done in various DENV prone parts of India. Moreover since human infection with one serotype provides lifelong Immunity to the individual against that specific serotype, but does not provide immunity against other serotypes.

On the contrary, since, cross-reactive antibodies enhances the severity of disease caused by subsequent infection by other serotype, thus, in dengue endemic countries, further research is needed to determine the patients immunological status prior to implementation of vaccination with tetra valent vaccine in endemic areas.

\section{Author's contribution}

Dr Nidhi Prasad was the principal investigator who gave significant in put in the development of this article. Dr Vidyut and Dr Keshav were involved in analyzing the data and completion of this manuscript. All authors read and approved the final manuscript.

\section{Acknowledgements}

We acknowledge all the staff members of IGIMS virology lab for the proper documentation of reports and for their technical support during data collection.

\section{Financial support}

There was no financial support. 


\section{References}

Arunachalam N, Murty US, Kabilan L et al., Studies on dengue in rural areas of Kurnool District, Andhra Pradesh, India. JAmMosqControl Assoc 2004; 20: 87-90

Bhatt S, Gething PW, Brady OJ, Messina JP, Farlow AW, Moyes CL et al., The global distribution and burden of dengue. Nature; 496: 504-507.

Brady OJ, et al., Refining the global spatiallimits of dengue virus transmission by evidence-based consensus. PLoSNegl TropDis. 2012; 6: e1760. doi:10.1371/journal.pntd. 0001760.

Brady OJ, Gething PW, Bhatt S, Messina JP, Brownstein JS, Hoen AG et al., Refining the global spatial limits of dengue virus transmission by evidence-based consensus. PLoSNeglTropDis. 2012; 6: e1760. doi:10.1371/journal. pntd.000 1760.

Chakravarti A, Arora R, Luxemburger C. Fifty years of dengue in India. Trans RSocTropMedHyg 2012; 106: 273-282.

Dengue: Guidelines for Diagnosis, Treatment, Prevention and Control: New Edition Geneva: World Health Organization; 2009.

Fields virology, $5^{\text {th }}$ edition, page no-1110-1112. George S, and Soman RS. Studies on Dengue in Bangalore City: Isolation of virus from Man and Mosquitoes. Indian J MedRes. 1975; 63: 396-401.

Gubler DJ. Dengue and dengue hemorrhagic fever. Clin Microbiol Rev 1998; 11: 480 496

Gupta E, Dar L, Narang P, Srivastava VK, Broor S. Sero diagnosis of dengue during an outbreak at a tertiary care hospital in
Delhi. Indian J MedRes. 2005; 121: 36-8. Halstead, S.B., "Dengue," The Lancet, vol. 370, no. 9599, pp. 1644-1652, 2007.

Kaur H, Prabhakar H, Mathew P, Marshalla R, Arya M. Dengue haemorrhagic fever outbreak in October-November 1996 in Ludhiana, Punjab, India. Indian $\mathbf{J}$ MedRes. 1997; 106: 1-3.

Martina BE, Koraka P, Osterhaus A. Dengue Virus Pathogenesis: an Integrated View. Clin Microbiol Rev. 2009; 22: 564-581.

Mehendale SM, Risbud AR, Rao JA, Banerjee $K$. Outbreak of Dengue fever in rural areas of Parbhani district of Maharashtra, India. Indian J MedRes. 1991; 93: 6-11.

Padbidri VS, Mahadev PV, Thakre JP, Pant U, Illkal MA, Varghese GG, et al., Virological and entomological investigations of an outbreak of Dengue fever in Dhule district, Maharashtra. Indian J MedMicrobiol. 1996; 14: 25-32.

Robinson ML et al., Vector-Borne Disease is a Common Cause of Hospitalized Febrile Illness in India. AmJ TropMedHyg. 2018 Mar 26. doi:10.4269/ajtmh.17-0571.

Rodrigues FM, Patankar MR, Banerjee K, Bhatt PN, Goverdhan MK, Pavri KM, et al., Etiology of the 1965 epidemic of febrile illness in Nagpur City, Maharashtra state, India. Bull WHO. 1972; 46: 173-9.

Shrivastava S, Tiraki D, Diwan A, Lalwani SK, Modak M, Mishra AC, et al., (2018) Cocirculation of all the four dengue virus serotypes and detection of an ovel clade of DENV-4 (genotype I) virus in Pune, India during 2016 season. PLoSONE 13(2): e0192672. https://doi.org/10.1371/ journal.pone.0192672

WHO, 2018 Factsheet

\section{How to cite this article:}

Nidhi Prasad, Vidyut Prakash, Keshav Kumar Bimal and Shahi, S.K. 2018. Seroprevalence of Dengue Infection in Patients Suffering from Acute Febrile Illness in a Tertiary Care Centre-Time to Introduce Dengue Vaccine CYD-TDV to Susceptible Population. Int.J.Curr.Microbiol.App.Sci. 7(06): 1079-1085. doi: https://doi.org/10.20546/ijcmas.2018.706.128 